\title{
Human leukocyte antigen-E protein is overexpressed in primary human colorectal cancer
}

\author{
ESTRELLA MARIEL LEVY ${ }^{1,2}$, MICHELE BIANCHINI ${ }^{1}$, ERIKA MARÍA VON EUW ${ }^{3}$, MARIA MARCELA BARRIO $^{1}$, \\ ALICIA INÉS BRAVO ${ }^{4}$, DAVID FURMAN ${ }^{1}$, ENZO DOMENICHINI ${ }^{5}$, CARLOS MACAGNO ${ }^{2}$, \\ VICTOR PINSKY ${ }^{2,6}$, CINZIA ZUCCHINI $^{7}$, LUISA VALVASSORI ${ }^{7}$ and JOSÉ MORDOH ${ }^{1,3}$ \\ ${ }^{1}$ Centro de Investigaciones Oncológicas (CIO-FUCA); ${ }^{2}$ Maestria en Biología Molecular Médica, Universidad de Buenos Aires; \\ ${ }^{3}$ Laboratorio de Cancerología, Fundación Instituto Leloir, IIBA-CONICET; ${ }^{4}$ Unidad de Inmunopatología Molecular, \\ Hospital Eva Peron; ${ }^{5}$ Servicio de Patología, Instituto Alexander Fleming; ${ }^{6}$ Hospital M.B. de Martinez, Buenos Aires, \\ Argentina; ${ }^{7}$ Dipartimento di Istologia, Embriologia e Biologia Applicata, Università di Bologna, Italy
}

Received August 13, 2007; Accepted October 18, 2007

\begin{abstract}
HLA-E is a non-classical MHC molecule whose expression by tumour cells has been recently reported in several human cancer types. We studied HLA-E expression in colorectal cancer patients, its clinical significance and prognostic value, as well as characterized its expression in colorectal cancer cell lines. We analysed HLA-E expression at the transcript level by qRT-PCR in micro-dissected samples and at the protein level by semiquantitative immunohistochemistry on paraffin-embedded tissue sections from 42 biopsies of colorectal cancer patients. We observed that HLA-E transcript and protein are spontaneously overexpressed in a significant proportion of colorectal tumour biopsies, as compared to normal mucosae. We also found a negative correlation between HLA-E expression and the $\mathrm{CD}_{57}{ }^{+}$cells infiltrate. Moreover, we analysed HLA-E expression in several colorectal cancer cell lines and demonstrated that IFN- $\gamma$ upregulates the expression of membrane HLA-E in vitro. Interestingly, we demonstrated that colorectal cancer cell lines overexpressing HLA-E at the cell surface inhibited NK-mediated cell lysis. Although IFN- $\gamma$ regulatory role needs further investigation, we provide evidence suggesting that this cytokine, within the tumour microenvironment, could promote HLA-E translocation to the surface of tumour epithelial cells. Furthermore, we showed that upregulation of HLA-E could be a marker of shorter disease-free survival in Dukes' $\mathrm{C}$ patients and we
\end{abstract}

Correspondence to: Dr José Mordoh, Laboratorio de Cancerología, Fundación Instituto Leloir, IIBA-CONICET, Av. Patricias Argentinas 435, Buenos Aires, Argentina

E-mail: jmordoh@leloir.org.ar

Key words: colorectal cancer, HLA-E, immune escape suggest that this molecule renders tumours less susceptible to immune attack.

\section{Introduction}

Colorectal carcinoma (CRC) is the fourth most commonly diagnosed cancer and accounts for $10-15 \%$ of deaths from cancer in Western countries (1). After radical surgery for locally advanced primary tumours (Dukes' B and C), 5 - FUbased adjuvant chemotherapy and radiation therapy significantly improves overall survival rate, but at 5 years, 40 to $50 \%$ of patients will still die of the disease $(2,3)$. Virtually all patients with metastatic stage IV CRC will die of their cancer. Toxicity and lack of tumour specificity are the most important limits of conventional approaches, such as chemotherapy and radiation therapy (4). Investigators are therefore seeking novel therapeutic options such as immunotherapy, which exploits the naturally occurring defence mechanism, and is capable of evoking tumour-specific immune responses (5). The availability of new biologic agents such as Cetuximab in the treatment of patients with metastatic disease, has led to improved survival (6). Cetuximab, a Mab targeting epidermal growth factor receptor (EGFR), has shown efficacy as thirdline therapy and promising results in first-line phase II studies (7). This antibody can activate the immune system, evoking the antibody-dependent cellular cytotoxicity (ADCC) that appears to be one of the most important immune effector functions (8). The immune system can recognize and destroy nascent transformed cells, although occasionally 'altered' cells may avoid the immune-surveillance leading to clinical manifestations of cancer (9). Many components of the immune system are involved in this process, especially cytotoxic lymphocytes (CTL) and natural killer (NK) cells $(10,11)$. MHC class I molecules play a crucial role in immune responsiveness by binding peptides and presenting them to CTL. In humans, the classical MHC class Ia glycoproteins, HLA$\mathrm{A},-\mathrm{B}$ and $-\mathrm{C}$, are expressed on the surface of nearly all nucleated cells $(12,13)$. On the other hand, non-polymorphic MHC class Ib molecules, HLA-E, -F and -G, have elusive 
functions since, in addition to Ag presentation, these molecules are involved in immune regulation (14). In particular, HLA-E expression by target cells results in inhibition of the lytic process induced by NK $(15,16)$ and natural killer T (NKT) cells (17). HLA-E recognition and inhibition are mediated primarily through the CD94/NKG2A heterodimer, identified as a cell surface Ag expressed by most NK and NKT cells. Significant HLA-E expression has been mainly documented in cell lines derived from histiocytic lymphoma, melanoma, pancreatic and cervical carcinoma (18). Recently, HLA-E expression has been also reported in primary glioma (19) and melanoma (20). As previously reported by us, preliminary data of gene expression profiles indicated that HLA-E transcript was significantly increased in most of the analysed CRC specimens (21). However, HLA-E expression in CRC patients needs to be better characterized and its clinical significance and prognostic value investigated. Therefore, we studied at the transcript and protein levels, HLA-E expression in tumour samples of CRC stages Dukes' $\mathrm{B}$ and $\mathrm{C}$, and its correlation with disease-free survival and lymphocyte infiltration. We also analysed HLA-E expression in several CRC cell lines, to investigate its inhibitory effect on NK cells attack, which might enable CRC cells to evade immune control.

\section{Materials and methods}

Cell lines. Five CRC cell lines were obtained from the American Tissue Culture Collection (ATCC): HCT-116, Caco-2, HT-29, DLD-1 and T84 (derived from lung metastasis). All cell lines were grown at $37^{\circ} \mathrm{C}$ in a humid atmosphere containing $5 \% \mathrm{CO}_{2}$ with Dulbecco's modified Eagle's medium (DMEM, Invitrogen Life Technology), supplemented with $10 \%$ heat-inactivated fetal calf serum, $2 \mathrm{mM}$ L-glutamine, $3.5 \mathrm{mg} / \mathrm{ml}$ sodium carbonate and $4.5 \mathrm{mg} / \mathrm{ml}$ glucose (Colon Medium).

Cell line treatments. To induce HLA-E membrane expression, cell lines were treated with $250 \mathrm{U} / \mathrm{ml}$ human recombinant Interferon $\gamma$ (hr-IFN- $\gamma$ Imukin-Boehringer Ingelhem) for $16 \mathrm{~h}$. To enhance HLA-E surface expression, $12 \mu \mathrm{g} / \mathrm{ml}$ recombinant hu- $\beta_{2}$-microglobulin (BD Biosciences, Pharmingen) were added to $10^{5}$ colon cancer cells in each case and kept for $16 \mathrm{~h}$ in $300 \mu \mathrm{l}$ of colon medium at $37^{\circ} \mathrm{C}$.

Immunofluorescence analysis by FACS. Indirect immunofluorescence was performed on CRC cell lines using a specific mouse anti-human HLA-E monoclonal antibody (clone MEM-E/08, Abcam) and HLA-A,B,C (clone W6/32, BD Biosciences, Pharmingen) and $\beta_{2}$-microglobulin $\left(\beta_{2} \mathrm{~m}\right)$ (clone B2m-01, BioVendor); as a negative control we used

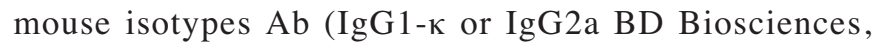
Pharmingen). Primary antibodies were incubated for $45 \mathrm{~min}$ at $4^{\circ} \mathrm{C}$. After washing, cells were incubated for $30 \mathrm{~min}$ with the secondary PE or FITC-labeled Mab (Dako Cytomation) and analyzed on a FACSCalibur flow cytometer using CellQuest software (BD Biosciences, Pharmingen) for data analysis. Intensity was calculated by the following formula: mean intensity level $(\mathrm{MIL})=[\%$ positive cells $] \times[$ mean fluorescence intensity].
For detection of intracellular HLA-E, washes and reagent dilutions were made with $1 \mathrm{X}$ PBS containing $0.1 \%$ saponin.

Western blot analysis of HLA-E. To perform Western blot analysis we used monoclonal antibody MEM-E/02 (BioVendor Laboratory Medicine, Inc., Czech Republic) to HLA-E molecule. Supernatants and cell lysates were collected after centrifugation for $5 \mathrm{~min}$ at $14,000 \mathrm{rpm}$ or 1,500 rpm respectively. Homogenization of cells was carried out in $0.1 \mathrm{ml}$ of lysis buffer (50 mM Tris- $\mathrm{HCl}, \mathrm{pH} 8.0,150 \mathrm{mM}$ $\mathrm{NaCl}, 0.1 \%$ SDS, $0.5 \%$ DCS, $1 \%$ NP-40, 5 mM EDTA) containing a protease inhibitor cocktail for mammalian tissues (Sigma, USA). Protein lysates were supplemented with $4 \mathrm{X}$ sample buffer (250 mM Tris- $\mathrm{HCl}, \mathrm{pH} 6.8,0.8 \%$ SDS, $40 \%$ glycerol, 0.7 M 2- 3 mercaptoetanol) and heated for $5 \mathrm{~min}$ at $95^{\circ} \mathrm{C}$; equal amounts of total protein lysate from either tumour tissues or normal colonic mucosa were loaded in each lane and separated in $12 \%$ SDS-PAGE; proteins were then electroblotted onto nitrocellulose membranes of $0.45-\mu \mathrm{m}$ pore size and the membranes blocked by incubation with PBS containing $0.2 \%$ Tween and 5\% BSA (bovine serum albumin). Following homogenization, protein lysates were quantified by Bradford method (22), separated by electrophoresis and finally transferred onto nitrocellulose membranes. The membranes were probed with anti-HLA-E antibody (diluted 1:3,000) for $1 \mathrm{~h}$ at room temperature. After washing, membranes were incubated for $45 \mathrm{~min}$ at room temperature with goat anti-mouse alkaline phosphatase (Jackson Immunoresearch Lab. Inc., USA) and washed thoroughly. Signals were detected using BCIP/NBT colour development substrate (Promega, USA).

Calcein cytotoxicity assay. DLD-1 cell line, pretreated or not with recombinant human $\beta_{2}$-microglobulin $\left(r h u-\beta_{2} \mathrm{~m}\right)$, was used as target $(\mathrm{T})$ and labeled with calcein-acetyoxymethyl (Calcein-AM) purchased from Molecular Probes (Invitrogen Life Technology) as a $1 \mathrm{mg} / \mathrm{ml}$ solution in dry dimethyl sulfoxide. Effector NK cells (E) were obtained from total lymphocytes separated from peripheral blood of healthy human volunteers by density gradient centrifugation, and further purified using NK Cell Isolation Kit (Miltenyi Biotec, USA). Target cells were resuspended in complete colon medium at a final concentration of $10^{6} / \mathrm{ml}$ and incubated with $10 \mu \mathrm{M}$ calcein-AM for $30 \mathrm{~min}$ at $37^{\circ} \mathrm{C}$ with occasional shaking. After two washes in AIM V ${ }^{\circledR}$ Medium the cytotoxicity assay was performed using DLD-1 cells with different E:T ratios ranging from 100:1 to 10:1, in triplicate, and with three replicate wells for spontaneous (only target cells in AIM V Medium) and maximum release (only target cells in medium plus $1 \%$ Triton X-100) in a total volume of $150 \mu 1$. After incubation at $37^{\circ} \mathrm{C}$ in $5 \% \mathrm{CO}_{2}$ for $4 \mathrm{~h}, 125 \mu \mathrm{l}$ of each supernatant was harvested and transferred into new tubes. Samples were measured using a Luminescence Spectrometer (Thermo Spectronics AMINCO-Bowman ${ }^{\circledR}$ Series 2).

Patients and tissue samples. A total of 42 CRC tissues and 5 non-cancerous tissues were obtained from patients with sporadic CRC who underwent surgical resection at the J. Mendez Hospital, M.B. de Martinez Hospital and 
Table I. Primer sequences, amplicons length and primer concentrations for HLA-E and B-actin.

\begin{tabular}{|c|c|c|c|}
\hline Transcript & $\begin{array}{l}\text { Product } \\
\text { size (bp) }\end{array}$ & $\begin{array}{l}\text { Primer Conc. } \\
\quad(\mathrm{nmol} / \mathrm{l})\end{array}$ & Sequence \\
\hline \multirow[t]{2}{*}{ HLA-E } & 250 & 250 & 5'-GGGACACCGCACAGATTTT-3' \\
\hline & & 250 & 5'-CTCAGAGGCATCATTTGACTTTT-3' \\
\hline \multirow[t]{2}{*}{ ß-actin } & 103 & 250 & 5'-CACTCTTCCAGCCTTCCTTC-3' \\
\hline & & 250 & 5'-TACAGGTCTTTGCGGATGTC-3' \\
\hline
\end{tabular}

Instituto Alexander Fleming (Buenos Aires, Argentina). The Institutional Review Boards of these institutions approved this research. Informed consent was obtained from each patient. Each resected cancer specimen was evaluated for its tumour cell content in hematoxylin and eosin-stained sections.

Laser microdissection (LMD). Several surgical specimens of $\mathrm{CRC}$ and the corresponding normal mucosae were freshly obtained, immediately embedded in RNAlater ${ }^{\mathrm{TM}}$ (Qiagen, Hiden, Germany) overnight at $4^{\circ} \mathrm{C}$ and then washed with PBS (RNase free) before freezing at $-80^{\circ} \mathrm{C}$ in OCT (Optimal Cutting Temperature, Tissue-Tek) until further use. Sample sectioning was carried out with a cryostat (Leica, Germany), immediately placed on special slides for LMD [steel frames with PEN (polyethylene naphthalate), Leica, Germany], which are specifically designed to free the dissectate from the section. Prior to hematoxylin staining, air-dried sections were fixed with methacarn $(60 \% \mathrm{v} / \mathrm{v}$ absolute methanol, $30 \% \mathrm{v} / \mathrm{v}$ chloroform and $10 \% \mathrm{v} / \mathrm{v}$ glacial acetic acid) for $10 \mathrm{~min}$ at room temperature. For expression analysis, only normal or tumour epithelial cells were microdissected using a laser microdissector system (AS LMD, Leica, Germany); dissected cells were catapulted into caps of PCR vials containing lysis buffer for RNA extraction.

Real-time PCR for HLA-E quantification in cancer cells from CRC tissues. cDNAs were synthesized from RNA samples extracted using RNAqueous ${ }^{\circledR}$-Micro (with the upgrade for RNA isolation from LMD samples) from CRC frozen sections, microdissected as above described. DNA was digested with RNase-free DNase I for $30 \mathrm{~min}$ at $37^{\circ} \mathrm{C}$ using a DNA-free kit (Ambion Inc., USA) following digestion, DNase was inactivated and removed using DNase inactivation reagent included in the kit. Reverse transcription was performed for $50 \mathrm{~min}$ at $37^{\circ} \mathrm{C}$ using random hexamer primers and Superscript II reverse transcriptase (Invitrogen Life Technology). RT-PCR products were analysed in triple determinations for the expression of HLA-E transcript by quantitative real-time PCR. To check for variations in amounts of RNA, $\beta$-actin was also tested as housekeeping gene. All PCR reactions were performed in an ICycler $^{\circledR}$ from BioRad using Platinum Taq DNA Polymerase and SYBR Green (Invitrogen Life Technology). Amplification efficiency and error were estimated for each pair of primers by standard curve calculation (derived from serial dilutions of positive
cDNA). The primer sequences used in this study, amplicon sizes, annealing temperatures and primers concentrations are shown in Table I.

After amplification, the temperature was slowly raised above the melting point of the PCR product to measure the fluorescence for the melting curve (this facilitates the identification of specific transcripts). Expression levels of target genes were referred relative to the expression levels of $\beta$-actin.

Immunohistochemistry (IHC) analysis. Mab to HLA-E (clone MEM-E/02, BioVendor Laboratory Medicine) was applied on colon tissue formalin fixed paraffin-embedded sections, using an indirect immunostaining technique. In brief, sections were deparaffinized and hydrated, endogenous peroxidase activity was quenched by incubation with $2 \% \mathrm{H}_{2} \mathrm{O}_{2}$ in methanol for $10 \mathrm{~min}$, microwave pretreatment in $10 \mathrm{mM}$ citrate buffer $\mathrm{pH} 6.0$ was employed to retrieve antigenicity for MEM-E/02 that reacts specifically with the denatured heavy chain of human HLA-E. Rehydratated sections were incubated overnight at $4^{\circ} \mathrm{C}$ with primary antibody using the optimal dilution $(1: 200)$ and for $30 \mathrm{~min}$ at room temperature with biotinylated anti-mouse $\mathrm{IgG}$ diluted in $1 \mathrm{X}$ PBS $0.2 \%$ Tween-20. The immunostaining reaction was developed using VectaStain ${ }^{\circledR}$ ABC Reagent and NovaRed ${ }^{\text {TM }}$ substrate solution (Vector Laboratories Inc., USA). The same histological slides were also probed for $\mathrm{CD} 57^{+}$cells using a monoclonal antibody (mouse anti-CD57, clone NK-1, Zymed Laboratories Inc., USA). The immunostaining reaction was developed using the LSAB substrate (Dako, Denmark). Sections were finally counterstained with hematoxylin, dehydrated and permanently mounted in nonaqueous mounting media.

Evaluation of tissue immunostaining. Immunohistochemistry was evaluated by three observers (E.L., A.I.B. and M.B.). IHC analysis of HLA-E expression and CD57+ infiltration in $\mathrm{CRC}$ and normal mucosa were performed by evaluating the percentage of positive cells in at least ten random fields (x400 magnification) using an optical light microscope (Olympus BX40, Japan). HLA-E staining was scored positive when CRC cells exhibited strong positivity as compared to PBS (cytoplasmic or membrane staining). A group with high and low HLA-E expression was distinguished based on 75th percentile of HLA-E expression scores of all analysed tumour tissues. 
A

HLA-E perm
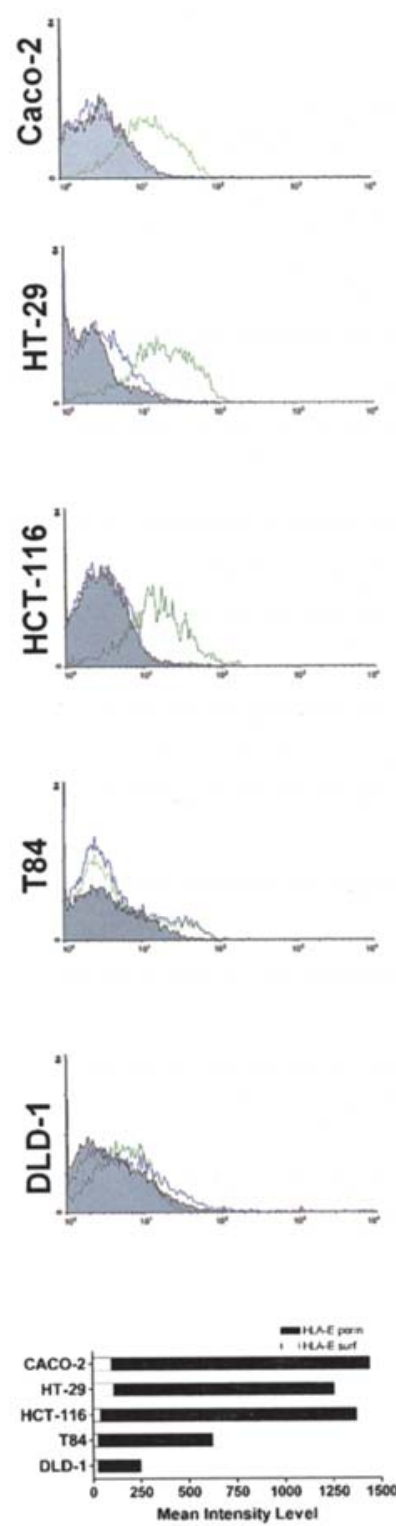

B

HLA-E + IFN- $\gamma$
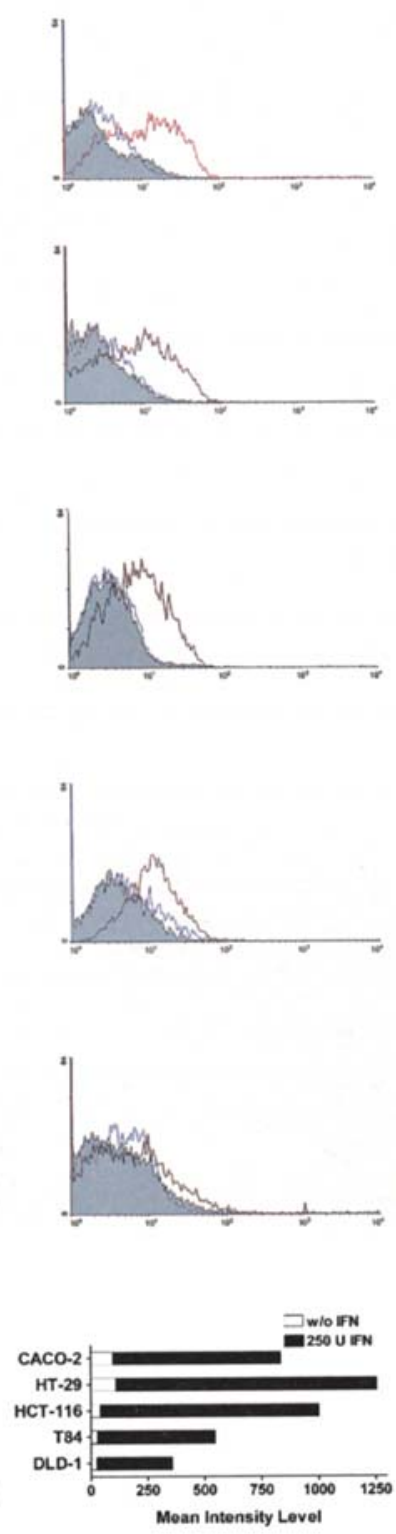

C HLA-Ia
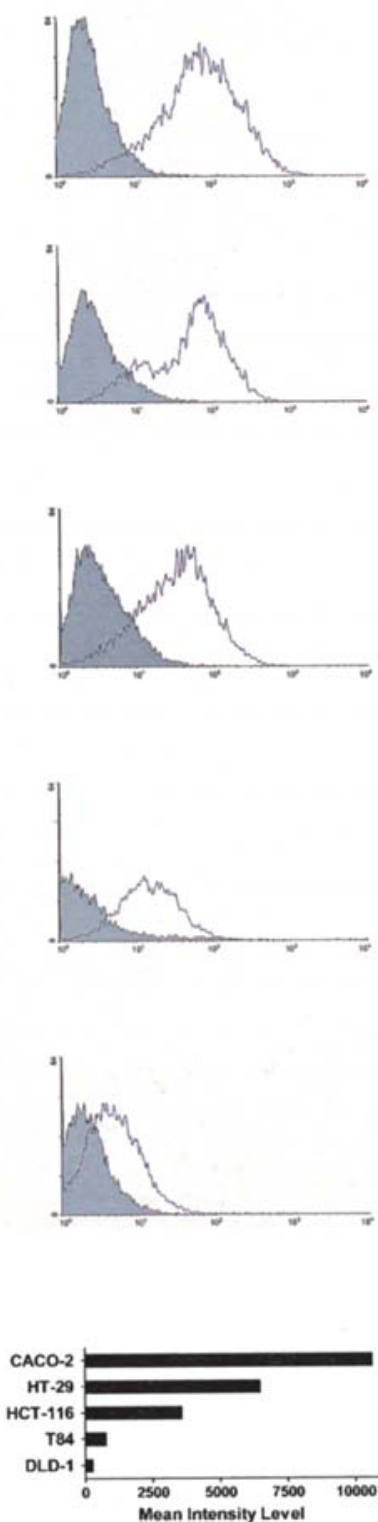

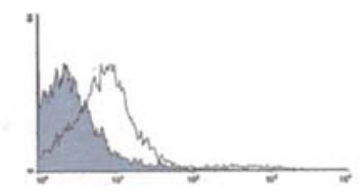

\section{$\beta 2$-microglobulin}
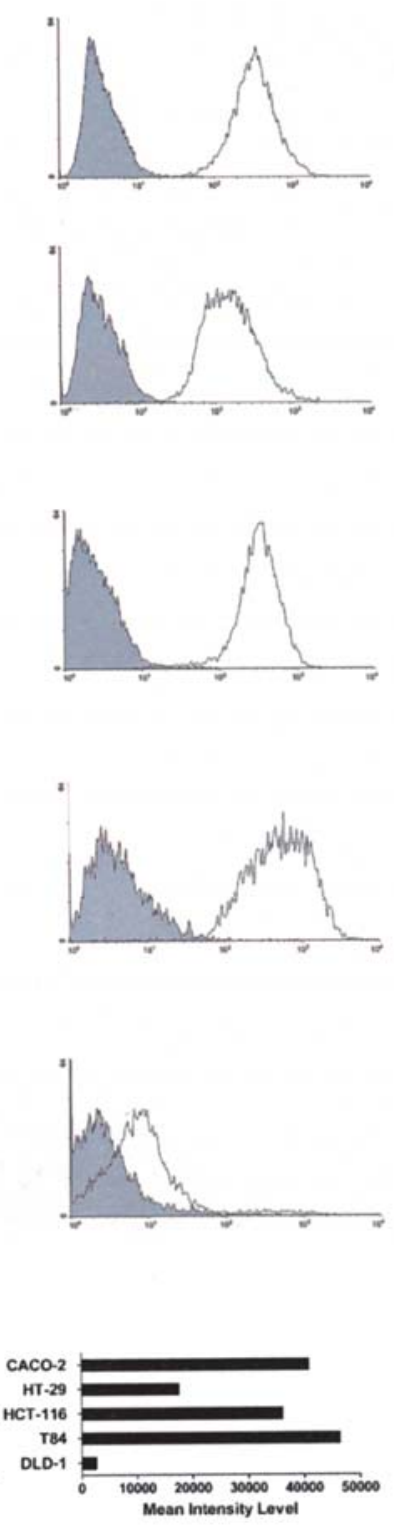

Figure 1. In vitro HLA-E expression by CRC cell lines. (A) Flow cytometry profiles for membrane (blue profiles) and intracellular (green profiles) HLA-E expression of five colorectal cell lines permeabilised by $0.1 \%$ saponin. (B) Flow cytometry profiles for HLA-E cell surface expression of five colorectal cell lines treated (red profiles) or not (blue profiles) with IFN- $\gamma$. (C) Flow cytometry profiles for HLA-Ia (clone W6/32) cell surface expression of five colorectal cell lines (blue profiles). (D) Flow cytometry profiles for $\beta_{2} \mathrm{~m}$ cell surface expression of five colorectal cell lines (blue profiles). Bar graphs at the bottom of the figure represent MIL (mean intensity level) that is calculated as follows: $(\mathrm{MIL})=(\%$ positive cells) $\mathrm{x}$ (mean fluorescence intensity).

Statistical analysis. Statistical analysis of the data was performed using GraphPad Prism v4.0. Patient characteristics, HLA-E expression and CD57+ infiltration were summarized using mean, standard deviation and median. Analysis of significance was performed using the 2-tailed Student's t-test with Welch's correction; $\mathrm{P}<0.05$ was considered significant and the $\mathrm{P}<0.01$ highly significant. Disease-free survival curves for CRC patients with high or low HLA-E expression levels were constructed using the Kaplan-Meier method. The log-rank test was used to compare the equality of the two curves. Contingency test was used to examine the relationship between HLA-E expression and tumour stage (Dukes' B and C). Signification was evaluated using a $\chi^{2}$-test with one degree of freedom; $\mathrm{P}<0.05$ was considered significant.

\section{Results}

HLA-E translocation to the cell surface is mediated by IFN- $\gamma$. As previously reported by us (21), mRNA expression profile of 25 primary human CRC tissues indicated that HLA-E was one of the most overexpressed transcripts in CRC tumours as compared to normal epithelium. As a continuation, and in order to determine its subcellular localization, we studied HLA-E expression in several human CRC cell lines (HT-29, HCT-116, Caco2, DLD-1 and T84). Less than five percent 

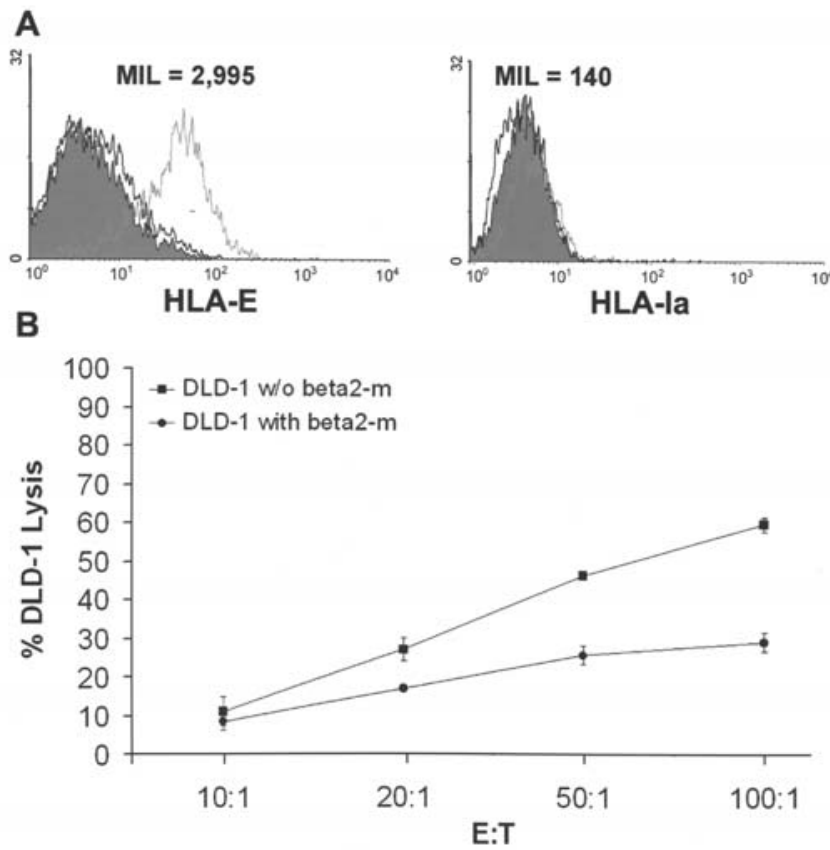

Figure 2. (A) Stabilization of cell surface expression of the HLA-E (left) and HLA-Ia (right) molecules by excess $r h u-\beta_{2} \mathrm{~m}$ in the culture medium. DLD-1 cell line was cultured in presence (left-light grey profile) or not (left-black profile) of $r h u-\beta_{2} \mathrm{~m}$ and subsequently stained with mAb against HLA-E (MEM-E/08). Similarly, DLD-1 cell line was cultured in presence (rightlight grey profile) or not (right-black profile) of $r h u-\beta_{2} \mathrm{~m}$ and subsequently stained with mAb against HLA-Ia (W6/32). In all cases cells were stained with isotype control (filled grey profiles) as negative control. (B) Consequence of membrane HLA-E expression by colorectal cancer cells (DLD-1) on their susceptibility to NK lysis. DLD-1 cells treated (square) or untreated (triangle) with $r h u-\beta_{2} \mathrm{~m}$ for $16 \mathrm{~h}$, were used as targets. The experiment was performed in triplicate using four different effector:target (NK:DLD-1) ratios. Colon cancer cells lysis was evaluated by measuring fluorescence of calcein-AM released in the medium.

of HLA-E cell surface expression was detected in all cell lines as shown by MIL calculation (average MIL=173 SE \pm 43) (Fig. 1 A). To investigate if HLA-E was retained intracellularly, cells were permeabilised using saponin. As shown in Fig. 1A, HLA-E is spontaneously highly expressed in $3 / 5$ cell lines analysed (average $\mathrm{MIL}=615 \mathrm{SE} \pm 179$ ), and HLA-E surface expression was significantly increased in $4 / 5$ cell lines (except DLD-1) upon IFN- $\gamma$ treatment (average $\mathrm{MIL}=807 \mathrm{SE} \pm 155$ ) (Fig. 1B). We also analysed HLA-Ia and $\beta_{2} \mathrm{~m}$ expressions with $\mathrm{W} 6 / 32$ and $\mathrm{B} 2 \mathrm{M}-01$ monoclonal antibodies, respectively. In contrast to HLA-E, these molecules were spontaneously expressed at significantly higher levels at the cell surface in 4/5 colorectal cell lines (except DLD-1) (average HLA-Ia MIL $=3,519 \mathrm{SE} \pm 1,195$; average $\left.\beta_{2} \mathrm{~m} \mathrm{MIL}=24,410 \mathrm{SE} \pm 7,795\right)($ Fig. $1 \mathrm{C}$ and D). Reduced expression of $\beta_{2} \mathrm{~m}$ in DLD-1 cell line correlates with a mutation in one allele of the $\beta_{2} \mathrm{~m}$ gene (23). This alteration would explain the lack of recognition of HLA-Ia and HLA-E in this cell line, either spontaneously or after IFN- $\gamma$ treatment, since monoclonal antibodies W6/32 and MEM-E/08 only recognize their targets when they are complexed with $\beta_{2} \mathrm{~m}$ in their native form.

rhu- $\beta_{2} m$ induces surface expression of HLA-E in DLD-1 cells which represent a useful model to analyse susceptibility to
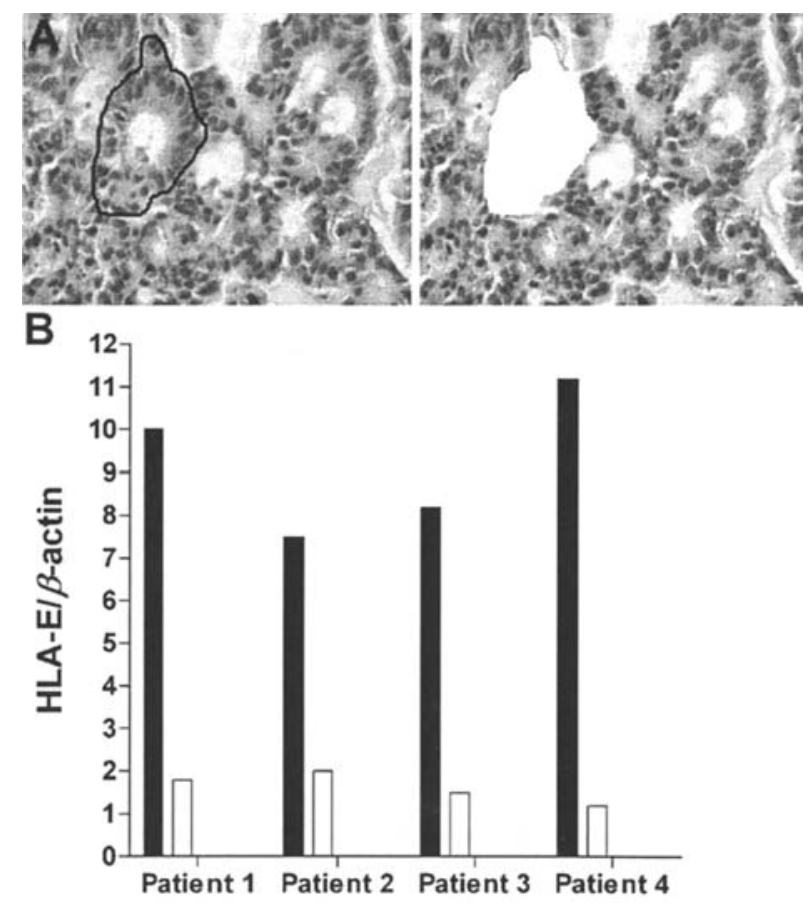

Figure 3. Representative pictures of microdissected areas from cryopreserved colorectal cancer samples; microdissection was used to selectively acquire epithelial colon cancer cells from both tumour and normal sections (A left panel, colon tissue before LMD; A right panel, colon tissue after LMD). (B) Between 200-400 ng of total RNA was extracted from microdissected epithelial cells in each case. HLA-E expression is reported as the ratio between its transcript level and $B$-actin level; black bars indicate tumour colon samples while white bars normal colon samples.

NK lysis. DLD-1 cells have a spontaneously diminished HLA-Ia and HLA-E surface expression because of $\beta_{2} \mathrm{~m}$ mutation; for this reason it was of interest to investigate if addition of $r h u-\beta_{2} \mathrm{~m}$ restored this diminished expression by stabilizing free heavy chains on the cell surface, as previously reported by others in a murine myeloma cell line (24). Surprisingly, upon incubation of DLD-1 cell line for $16 \mathrm{~h}$ in the presence of exogenous $r h u-\beta_{2} \mathrm{~m}$, only HLA-E cell surface expression was reconstituted $\left[\mathrm{MIL}_{(\mathrm{HLA}-\mathrm{E})}=2,995\right.$; $\mathrm{MIL}_{\text {(HLA-Ia) }}=140$ ] (Fig. 2A), thus offering a valuable model to dissect NK from CTL activity. Accordingly, we coincubated DLD-1 cells pre-treated or not with $r h u-\beta_{2} \mathrm{~m}$, with polyclonal NK cells from healthy donors during $4 \mathrm{~h}$. As a result, we observed that DLD-1 cells were strongly lysed by NK cells $(59.7 \% \pm 1.98)$, but when $r h u \beta_{2} \mathrm{~m}$ was added lysis was reduced $(29.3 \% \pm 2.44)$, thus directly demonstrating the protective effect of HLA-E (Fig. 2B). These results agree with previous studies $(19,20)$ showing that HLA-E expression at the cell membrane of cancer cells prevents NK lytic activity.

The HLA-E transcript is overexpressed in microdissected epithelium from frozen human CRC samples. By real-time PCR quantitative method, HLA-E mRNA expression was evaluated in epithelial colon cells from several pairs of frozen cancerous and normal colorectal samples from the same patient. Laser microdissection was used to specifically select both several tumour-containing areas and normal epithelium from the same patient (Fig. 3A). qRT-PCR assay demonstrated that HLA-E expression was increased in 
Table II. Clinical characteristics of CRC patients.

\begin{tabular}{|c|c|}
\hline Total patients & 42 \\
\hline Male & 26 \\
\hline Female & 16 \\
\hline \multicolumn{2}{|l|}{ Age (years) } \\
\hline Mean & 66 \\
\hline Range & $32-89$ \\
\hline \multicolumn{2}{|l|}{ Dukes } \\
\hline $\mathrm{B}$ & 18 \\
\hline $\mathrm{C}$ & 24 \\
\hline \multicolumn{2}{|c|}{ Tumour invasion } \\
\hline pT1 & 4 \\
\hline pT2 & 2 \\
\hline pT3 & 34 \\
\hline pT4 & 2 \\
\hline \multicolumn{2}{|c|}{ Lymph node metastasis } \\
\hline pNO & 18 \\
\hline $\mathrm{pN} 1$ & 12 \\
\hline $\mathrm{pN} 2$ & 12 \\
\hline \multicolumn{2}{|c|}{ Pathologic grade } \\
\hline Well & 12 \\
\hline Moderate & 27 \\
\hline Poor & 3 \\
\hline \multicolumn{2}{|l|}{ Location } \\
\hline Left & 12 \\
\hline Right & 23 \\
\hline Rectal & 7 \\
\hline
\end{tabular}

epithelial cells from tumour samples as compared with epithelia from normal mucosae (Fig. 3B).

HLA-E protein is overexpressed in human CRC samples. Our in vitro results suggest that once HLA-E is translocated to the cell surface of colon cancer cells, innate immune response may be compromised. Therefore, HLA-E expression in colorectal epithelium cells was investigated by IHC on sections of tumour samples from 42 patients (Table II).

In agreement with the results at the transcript level, strong protein expression of HLA-E was observed in tumour samples (Fig. 4B and C), while weak staining was detected in samples of normal mucosae (Fig. 4A). Infiltrating lymphocytes were used as HLA-E internal positive control. The mean percentage value for HLA-E positive cells in Dukes' $\mathrm{C}$ tumour samples was $72.4 \pm 3.1$; this percentage proved significantly different from Dukes' B tumour samples (mean 54.4 \pm 5.7 ) and normal mucosae samples (mean 6.2 \pm 1.2 ) (Fig. 4D).

HLA-E correlation with disease-free survival. According to our finding that HLA-E induced expression protects
DLD-1 from NK attack (Fig. 2B) we decided to analyse the correlation between HLA-E expression and disease-free survival. The forty-two patients were classified defining two groups (HHE, high HLA-E expression and LHE, low HLA-E expression) based on the 75 th percentile of the expression scores of all analysed tumour tissues (Fig. 5A). Comparison by the Kaplan-Meier method revealed a significant decrease in disease-free survival of patients belonging to the HHE group (log-rank $\mathrm{P}=0.0028$; Hazard Ratio $0.18 \%, 95 \% \mathrm{CI}$ of ratio 0.012-0.402) (Fig. 5B). However, applying $\chi^{2}$-test we determined that the level of HLA-E protein expression was not independent of tumour grade, since a high proportion of LHE expressing tumours belongs to the Dukes' B group (Dukes' B and C vs. HHE and LHE; $\mathrm{P}=0.002$ ). For this reason, we decided to study by Kaplan-Meier the disease-free survival for Dukes' C patients belonging either to the HHE and LHE groups, and we could determine that HHE group displayed a significant decrease in disease-free survival ( $\log$-rank $\mathrm{P}=0.0068$; Hazard Ratio $0.11 \%, 95 \% \mathrm{CI}$ of ratio 0.007-0.456) (Fig. 5C).

Correlation of lymphocyte infiltration in colorectal tumour samples with HLA-E expression. Thirty-seven CRC tumours were assessed histopathologically for immune-cell infiltrates within the tumour. $\mathrm{CD} 57^{+}$cells (largely representing NK cells) were analysed and counted in relation to either the stage of the tumours (Dukes' B and C) or HLA-E expression level (Fig. 6A and B). Immunostaining with CD57 antibody revealed that while there was not a significant difference between tumour stages (Dukes' B vs. Dukes' C; Fig. 6C), HHE tumours contained lower numbers of $\mathrm{CD} 57^{+}$cells as compared with LHE tumours (HHE vs. LHE; Fig. 6D).

Colon cancer cell lines produce a soluble form of HLA-E. The diminished $\mathrm{CD} 7^{+}$cell infiltrate observed in HHE group could be due to NK-induced apoptosis by the soluble form of HLA-E, as previously reported by others (25). In order to analyse if HLA-E might be produced in soluble form, we used another monoclonal antibody, MEM-E/02, which recognizes the denatured heavy chain of HLA-E (26). By Western blot, we were able to detect soluble HLA-E (sHLA-E) in culture supernatants from IFN- $\gamma$-activated CRC cells (Fig. 7). Soluble HLA-E was detected as a band of $37 \mathrm{kDa}$, corresponding to the metalloproteinase-dependent shedding of membrane-bound protein (27). Therefore, colon cancer cell activation by IFN- $\gamma$ results in both increased membrane-bound HLA-E expression (Fig. 1B) and release of sHLA-E.

\section{Discussion}

Colorectal cancer cells may avoid adaptative immune response by downregulating classical MHC-class I molecules. There is sufficient evidence that CRC cells frequently lose or underexpress MHC-class I (28). Paradoxically, MHC class I total loss correlates to a better outcome since such cells are strong targets for NK-cells attack (29). Based on our findings, we hypothesized that HLA-E surface expression in CRC cells could represent a complementary strategy to avoid innate surveillance, further contributing to immune escape. In this 

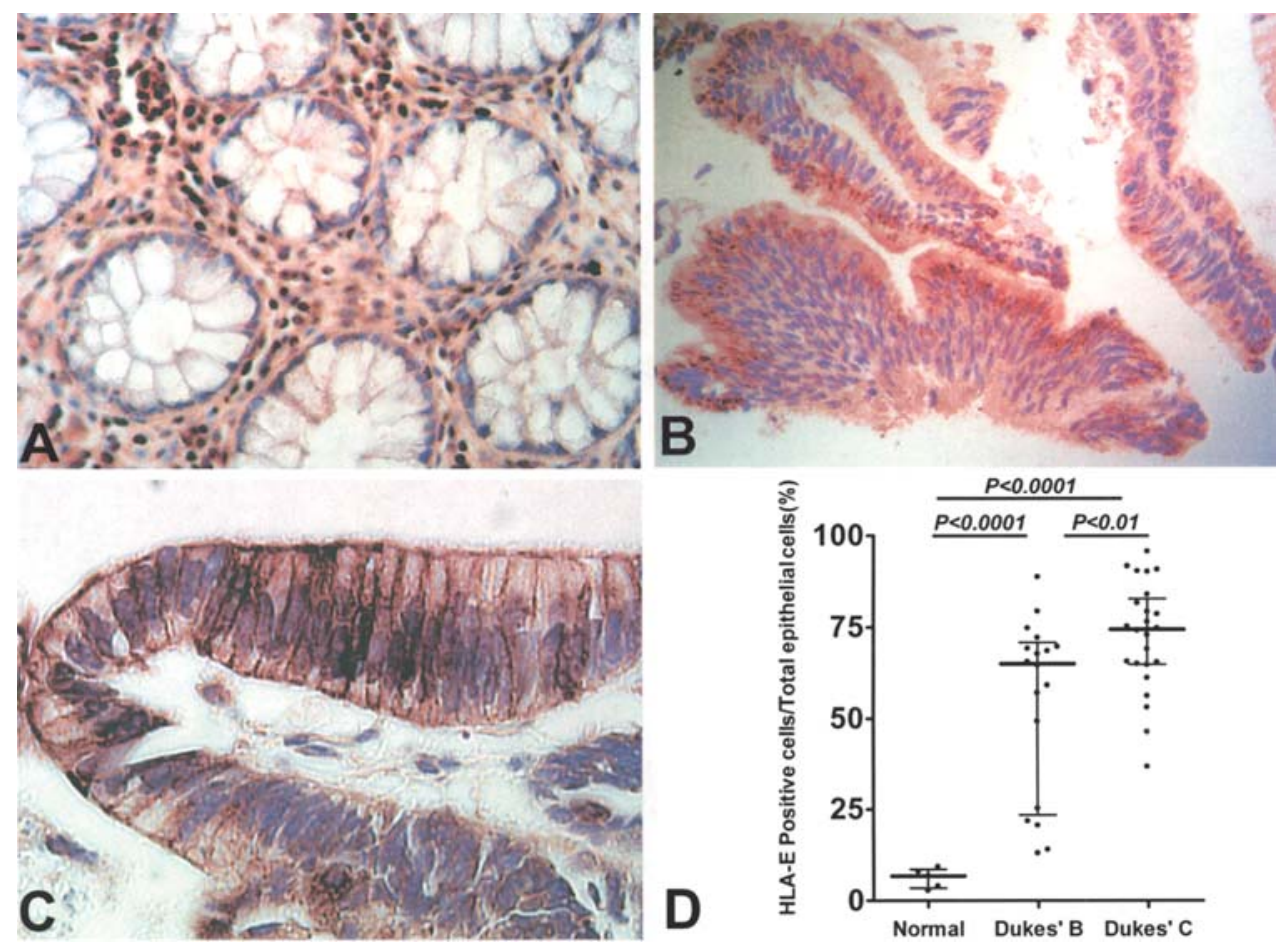

Figure 4. Representative photos of immunoperoxidase staining of surgically removed colorectal tissues. (A-C) HLA-E protein expression. (A) Low expression (1-15\% cells positive) in normal epithelium (infiltrating lymphocytes staining was used as positive control). (B) Medium expression prevalently at the intracellular level (16-75\% cells positive) usually observed in Dukes' B samples. (C) High expression prevalently at membrane level (76-100\% cells positive) in Dukes' C patients. (D) Dot plot graph of immunohistochemistry showing the percentage of HLA-E positive cells/total epithelial cells on different normal and tumour colon samples (median with interquartile range).
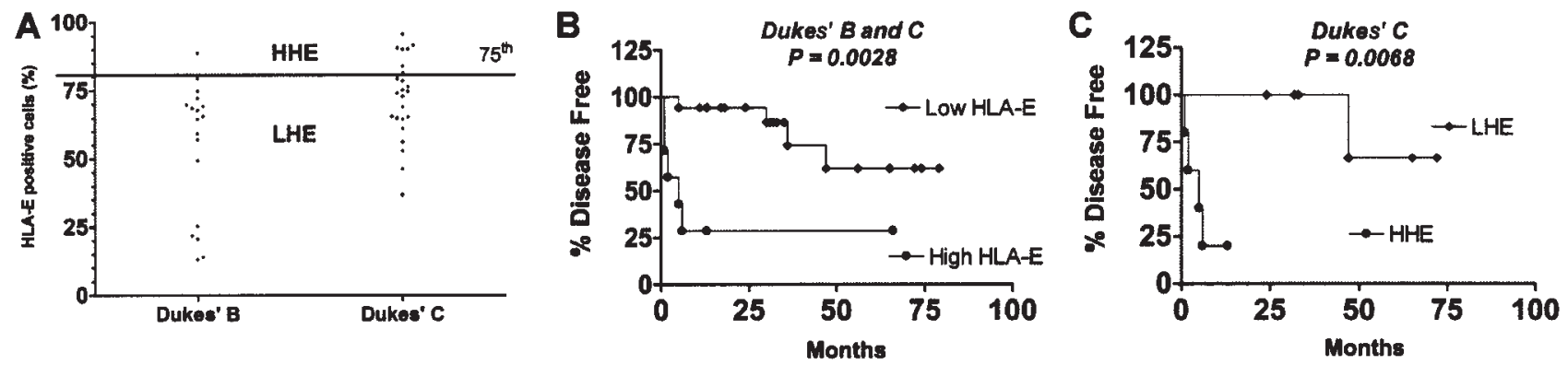

Figure 5. Correlation of HLA-E expression with disease free survival. (A) The graph shows HLA-E cut-off to define two groups of high or low HLA-E expression based on the 75th percentile of the expression scores of all analysed tumour tissues by immunohistochemistry. The 42 patients were classified into two groups (HHE, high HLA-E expression $\mathrm{n}=8$ and LHE, low HLA-E expression, $\mathrm{n}=34$ ). (B) Kaplan-Meier survival curves comparing disease-free survival between CRC patients with high and low HLA-E expression (log-rank P=0.0028; Hazard Ratio 0.18\%, 95\% CI of ratio 0.012-0.402). (C) KaplanMeier survival curves comparing disease-free survival between CRC patients belonging to Dukes' C stage with high and low HLA-E expression (logrank $\mathrm{P}=0.0068$; Hazard Ratio $0.11 \%, 95 \% \mathrm{CI}$ of ratio $0.007-0.456)$.

study we show for the first time that a significant fraction of CRC cells spontaneously overexpress HLA-E in tumour biopsies from CRC patients. This expression was first analysed in vitro on several metastatic colon cancer cell lines, showing that HLA-E cell surface expression is quite low, despite the presence of high levels of intracellular HLA-E protein. This finding suggests that most CRC cell lines produce significant amount of HLA-E molecules but the absence of T-cell cytokine in vitro could perhaps explain the lack of HLA-E surface expression (30). For this reason, we thought that the addition of a cytokine such as IFN- $\gamma$ could mimic the in vivo stimulation of HLA-E expression
$(20,31)$. In fact, using IFN- $\gamma$ we could induce HLA-E membrane expression and its release to the medium, suggesting that this cytokine could induce a rapid mobilization of HLA-E from intracellular stores. This mechanism could represent an important survival advantage for tumour cells, which could rapidly respond to immune attack by NK, in particular when HLA-Ia molecules are downregulated (32). Paradoxically, tumour cells could take advantage of IFN- $\gamma$ release that should render target cells more susceptible to CTL.

Nonetheless, the consequences of HLA-E translocation to cell membrane in CRC cells for innate immune escape have 


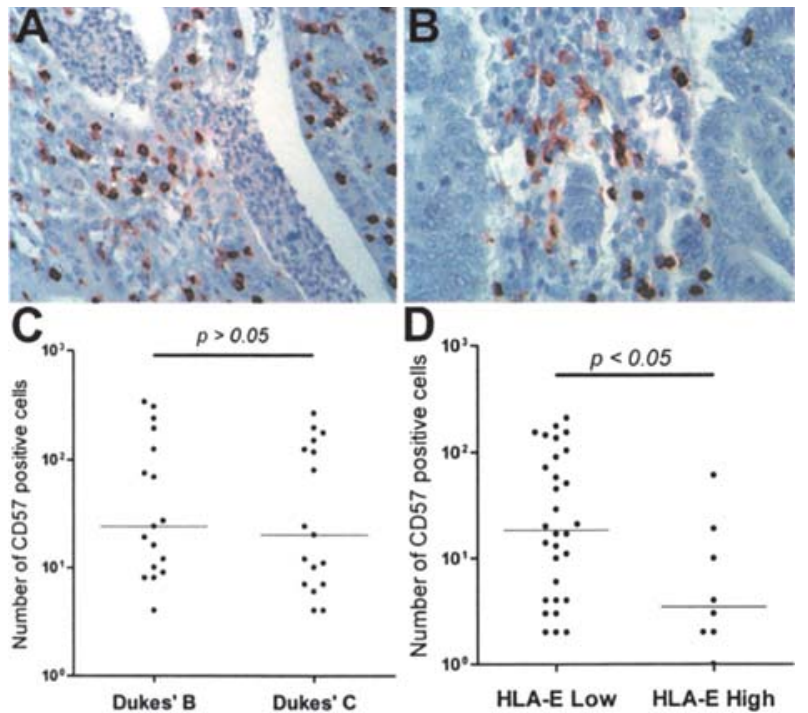

Figure 6. Infiltrating lymphocyte characterization using monoclonal antibody against $\mathrm{CD}^{+} 7^{+}$lymphocytes. Representative photos of infiltrating CD57 $7^{+}$cells in Dukes' B (A) and Dukes' C (B) tumours. Comparison of $\mathrm{CD}^{2} 7^{+}$cells in primary colorectal tumours showing Dukes' B vs. Dukes' C (C) and HHE vs. LHE (D). Staining with antibodies revealed that Dukes' B and $\mathrm{C}$ tumours contained similar number of $\mathrm{CD} 57^{+}$cells $(\mathrm{P}>0.05)$ while HHE tumours had significantly decreased infiltrating CD $57^{+}$lymphocytes $(\mathrm{P}<0.05)$

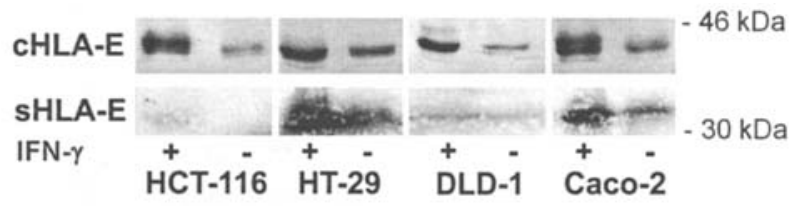

Figure 7. Detection of sHLA-E by Western blot analysis in culture supernatants and lysates of four colorectal cancer cell lines. The mechanism for the IFN- $\gamma$ induced generation of the sHLA-E was evaluated, as follows: CRC cell lines were incubated or not with IFN- $\gamma$ for $24 \mathrm{~h}$, culture supernatants and cells were analysed by SDS-PAGE, transferred to nitrocellulose membrane and probed with mAb MEM-E/02.

not yet been investigated. Since HLA-E could not be induced by IFN- $\gamma$ treatment of DLD- 1 cell line because of the $\beta_{2} \mathrm{~m}$ mutation, to demonstrate if HLA-E surface expression suppresses NK cell-mediated target lysis, we designed a model based on HLA-E structural stabilization on DLD-1 cells by exogenous $r h u-\beta_{2} \mathrm{~m}$ addition (23). Our data show that colon cancer cells expressing stable levels of membrane HLA-E are less susceptible to NK attack; this strategy utilizes a simple cellular model that avoids the use of specific CTL clones.

Taking into account these in vitro results, we decided to investigate in vivo the relevance of HLA-E expression using CRC tissues. We show for the first time that a significant fraction of CRC cells spontaneously overexpress HLA-E in vivo, as demonstrated at the transcript level by qRT-PCR and at the protein level by semiquantitative IHC on paraffinembedded tissue sections. IHC analysis of normal colon mucosae revealed only very few HLA-E positive epithelial cells, whereas a strong HLA-E expression was observed in tumour epithelial cells of different Dukes CRC grades,
HLA-E expression increasing with malignancy stage. The observation that HLA-E expression is higher in the Dukes' C stage of the disease suggests that in this group of patients tumour cells could escape the immune response by refractoriness to both infiltrating NK and NKT cells. Corroborating evidence for this hypothesis is provided by our finding that primary CRC expressing high levels of HLA-E protein display a significant decrease in $\mathrm{CD} 7^{+}$cell infiltration compared to low level HLA-E expressing tumours. We have chosen CD57 monoclonal antibody to identify NK cells because it recognizes a carbohydrate antigen present in a subset of peripheral blood mononuclear cells involved in NK activity in CRC (33-35) as well as in lung cancer (36). The reduction in $\mathrm{CD}_{57} 7^{+}$cell infiltration could be explained by the pro-apoptotic action reported by Spaggiari and coworkers (25) for soluble forms of HLA-I that, upon interaction with the activating receptor for HLA-I on the NK cell surface, may evoke NK cell apoptosis. Accordingly, we found that CRC cell lines released a soluble form of HLA-E. Interestingly, Dukes' $\mathrm{C}$ tumours with a high percentage of HLA-E positive cells $(>80 \%)$ were associated with shorter diseasefree survival than tumours with low percentage of HLA-E. These findings suggest that the survival benefit observed in tumours with low HLA-E expression may be due to killing of tumour cells by NK and NKT cells at the tumour site. Our results highlight the importance of innate immunity in CRC patients outcome, in addition to adaptative immunity already reported by other authors $(37,38)$. These results are particularly pertinent to the development of immunotherapeutic strategies for CRC, suggesting that early stage patients with low HLA-E expression may derive greater benefit from immunotherapy, as their NK cells are likely to remove remaining tumour cells after surgery.

In conclusion, we have shown in this study that primary human CRC spontaneously express significant levels of HLA-E molecules, intracellular or membrane bound. Membrane HLA-E expression by primary CRC cells might contribute to the escape of these tumours from immune surveillance, by inhibiting the lysis by NK and NKT cells. In this respect, we showed that, as compared with low HLA-E expressing tumours, high HLA-E expressing tumours contained significantly less $\mathrm{CD} 57^{+}$cells. We believe that our data justify further studies in a larger group of patients to establish if high HLA-E levels could be a marker of poor prognosis in primary CRC. Patients with low expression of HLA-E might be better candidates for antibody therapies for example Cetuximab, since they should be susceptible to NK killing by ADCC. This would be of particular relevance in Dukes' C patients who show a better correlation between HLA-E expression and poor disease-free survival.

\section{Acknowledgements}

This work was supported with funds from Fundación Sales, Fundación Mosoteguy, Fundación Cancer (FUCA), Agencia Nacional de Promoción Cientifica y Tecnológica (ANPCyT). J.M. is a member of CONICET, E.M.L. belongs to MBMM (University of Buenos Aires), M.B. is a postdoctoral fellow of CONICET, M.M.B. is fellow of the Fundación Sales. E.M.v.E. is a fellow of the University of Buenos Aires. We 
thank Dr A. Tomas, Dr P. Gonzalez (Hospital M.B. de Martinez), Dr Martha Endelman, Dr Porqueres (Hospital Julio Mendez) and Dr Mora Amat for collaboration with CRC collection. We thank Amorina Wunderlich and Marcela Castro for technical assistance. This paper is dedicated to the memory of Victor Pinsky, who died shortly after completion of this study.

\section{References}

1. Smith R, Cokkinides V and Eyre HJ: American Cancer Society guidelines for the early detection of cancer. J Clin Cancer 56 : 11-25, 2006.

2. Goldberg RM and Gill S: Recent phase III trials of fluorouracil, irinotecan, and oxaliplatin as chemotherapy for metastatic colorectal cancer. Cancer Chemother Pharmacol 54: P57, 2004.

3. Pasetto LM, Jirillo A, Iadicicco G, et al: FOLFOX vs. FOLFIRI: a comparison of regimens in the treatment of colorectal cancer metastases. Anticancer Res 25: 563-576, 2005.

4. Kelloff GJ, Lippman SM, Dannenberg AJ, et al: AACR Task force on cancer prevention. Progress in chemoprevention drug development: the promise of molecular biomarkers for prevention of intraepithelial neoplasia and cancer - A plan to move forward. Clin Cancer Res 12: 3661-3697, 2006.

5. Choudhury A, Mosolits S, Kokhaei P, et al: Clinical results of vaccine therapy for cancer: learning from history for improving the future. Adv Cancer Res 95: 147-202, 2006.

6. Arsene D, Galais MP, Bouhier-Leporrier K and Reimund JM: Recent developments in colorectal cancer treatment by monoclonal antibodies. Expert Opin Biol Ther 6: 1175-1192, 2006

7. Saltz LB, Meropol NJ, Loehrer MN, et al: Phase II trial of cetuximab in patients with refractory colorectal cancer that expresses the epidermal growth factor receptor. J Clin Oncol 22: 1201-1208, 2004.

8. Iannello A and Ahmad A: Role of antibody-dependent cellmediated cytotoxicity in the efficacy of therapeutic anti-cancer monoclonal antibodies. Cancer Metastasis Rev 24: 487-499, 2005.

9. Dunn GP, Old LJ and Schreiber RD: The three Es of cancer immunoediting. Annu Rev Immunol 22: 329-360, 2004.

10. Moretta A, Bottino C, Vitale M, et al: Receptors for HLA class-I molecules in human natural killer cells. Annu Rev Immunol 14: 619-648, 1996.

11. Moretta A, Biassoni R, Bottino C, et al: Major histocompatibility complex class I-specific receptors on human natural killer and T lymphocytes. Immunol Rev 155: 105-117, 1997.

12. York IA and Rock KL: Antigen processing and presentation by the class I major histocompatibility complex. Annu Rev Immunol 14: 369-396, 1996.

13. Van Kaer L: Major histocompatibility complex class I-restricted antigen processing and presentation. Tissue Antigens 60: 1-9, 2002 .

14. O'Callaghan CA: Molecular basis of human natural killer cell recognition of HLA-E (human leucocyte antigen-E) and its relevance to clearance of pathogen-infected and tumour cells. Clin Sci 99: 9-17, 2000.

15. Lee N, Llano M, Carretero M, et al: HLA-E is a major ligand for the natural killer inhibitory receptor CD94/NKG2A. Proc Natl Acad Sci USA 95: 5199-5204, 1998.

16. Borrego F, Ulbrecht M, Weiss EH, et al: Recognition of human histocompatibility leukocyte antigen (HLA)-E complexed with HLA class I signal sequence-derived peptides by CD94/NKG2 confers protection from natural killer cell-mediated lysis. J Exp Med 187: 813-818, 1998.

17. Braud VM, Allan DSJ and McMichael AJ: Functions of nonclassical MHC and non-MHC-encoded class I molecules. Curr Opin Immunol 11: 100-108, 1999.

18. Marin R, Ruiz-Cabello F, Pedrinaci S, et al: Analysis of HLA-E expression in human tumors. Immunogenetics 54: 767-775, 2003.
19. Wischhusen J, Friese MA, Mittelbronn M, et al: HLA-E protects glioma cells from NKG2D-mediated immune responses in vitro: implications for immune escape in vivo. Neuropathol Exp Neurol 64: 523-528, 2005.

20. Derre L, Corvaisier M, Charreau B, et al: Expression and release of HLA-E by melanoma cells and melanocytes: potential impact on the response of cytotoxic effector cells. J Immunol 177: 3100-3107, 2006.

21. Bianchini M, Levy E, Zucchini C, et al: Comparative study of gene expression by cDNA microarray in human colorectal cancer tissues and normal mucosa. Int J Oncol 29: 83-94, 2006.

22. Bradford MM: A rapid and sensitive method for the quantitation of microgram quantities of protein utilizing the principle of protein-dye binding. Anal Biochem 72: 248-254, 1976.

23. Bicknell DC, Rowan A and Bodmer WF: Beta 2-microglobulin gene mutations: a study of established colorectal cell lines and fresh tumors. Proc Natl Acad Sci USA 91: 4751-4755, 1994.

24. Ulbrecht M, Kellermann J, Johnson JP, et al: Impaired intracellular transport and cell surface expression of non-polymorphic HLA-E: evidence for inefficient peptide binding. J Exp Med 176: 1083-1090, 1992.

25. Spaggiari GM, Contini P, Dondero A, et al: Soluble HLA class I induces NK cell apoptosis upon the engagement of killeractivating HLA class I receptors through FasL-Fas interaction. Blood 100: 4098-4107, 2002.

26. Menier C, Saez B, Horejsi V, et al: Characterization of monoclonal antibodies recognizing HLA-G or HLA-E: new tools to analyze the expression of non-classical HLA class I molecules. Hum Immunol 64: 315-326, 2003.

27. Dong Y, Lieskovska J, Kedrin D, et al: Soluble non-classical HLA generated by the metalloproteinase pathway. Hum Immunol 64: 802-810, 2003.

28. Algarra I, Garcia-Lora A, Cabrera T, et al: The selection of tumor variants with altered expression of classical and nonclassical MHC class I molecules: implications for tumor immune escape. Cancer Immunol Immunother 53: 904-910, 2004.

29. Watson NF, Ramage JM, Madjd Z, et al: Immunosurveillance is active in colorectal cancer as downregulation but not complete loss of MHC class I expression correlates with a poor prognosis. Int J Cancer 118: 6-10, 2006.

30. Garcia-Lora A, Martinez M, Algarra I, et al: MHC class Ideficient metastatic tumor variants immunoselected by $\mathrm{T}$ lymphocytes originate from the coordinated downregulation of APM components. Int J Cancer 106: 521-527, 2003.

31. Gustafson KS and Ginder GD: Interferon-gamma induction of the human leukocyte antigen-E gene is mediated through binding of a complex containing STAT1alpha to a distinct interferongamma-responsive element. J Biol Chem 271: 20035-20046, 1996.

32. Rees RC and Mian S: Selective MHC expression in tumours modulates adaptive and innate antitumour responses. Cancer Immunol Immunother 48: 374-381, 1999.

33. Sandel MH, Speetjens FM, Menon AG, et al: Natural killer cells infiltrating colorectal cancer and MHC class I expression. Mol Immunol 42: 541-546, 2005.

34. Coca S, Perez-Piqueras J, Martinez D, et al: The prognostic significance of intratumoral natural killer cells in patients with colorectal carcinoma. Cancer 79: 2320-2328, 1997.

35. Okada T, Iiai T, Kawachi Y, et al: Origin of CD57+ T cells which increase at tumour sites in patients with colorectal cancer. Clin Exp Immunol 102: 1591-1566, 1995.

36. Villegas FR, Coca S, Villarrubia VG, et al: Prognostic significance of tumor infiltrating natural killer cells subset CD57 in patients with squamous cell lung cancer. Lung Cancer 35: 23-28, 2002.

37. Pages F, Berger A, Camus M, et al: Effector memory T cells, early metastasis and survival in colorectal cancer. $\mathrm{N}$ Engl $\mathrm{J}$ Med 353: 2654-2666, 2005.

38. Galon J, Costes A, Sanchez-Cabo F, et al: Type, density, and location of immune cells within human colorectal tumors predict clinical outcome. Science 313: 1960-1964, 2006. 